\title{
CONTRIBUIÇÃO PARA O ESTUDO DA ANESTESIA INTRAVENOSA NO CÃO, PELO "PENTOBARBITAL SÓDIO" (NEMBUTAL)
}

(CONTRIBUTION TO THE STUDY OF THE INTRAVENOUS ANESTHESIA

IN THE DOG, BY THE "PENTOBARBITAL SODIUM") (NEMBUTAL)

\section{Ernesto Antônio Matera \\ Assistente \\ Oswaldo A. Castrignano \\ Vcterinário-Interno}

O emprêgo dos barbituratos na anestesia dos pequenos animais, vem ocupando uma posição de destaque nestes últimos anos, pois os barbituratos oferecem uma grande margem de segurança, duração suficiente e anestesia cirúrgica perfeita em relação a outros anestésicos até então conhecidos.

Interessados nas questões de anestesia que preocupam o médico veterinario, e tendo já usado com resultados satisfatórios um dos elementos do grupo dos barbituratos: o pentotal (thionembutal), nos propusemos a descrever a aplicação de outro barbiturato, o pentobarbital (nembutal).

O pentobarbital ocupa na classificação mais aceita dos anestésicos do tipo dos barbituratos, dada por Fitch, WAters e TATUM a seguinte colocação:

1. ação longa (8-36 horas) : barbital, fenobarbital e neonal.

2. ação curta (3-6 horas): pentobarbital (nembutal) e pernoston.

3. ação ultra curta (30-45 minutos) : evipal e pentotal (thionembutal).

Portanto, o pentobarbital sódio é um barbiturato de ação curta, oferecendo entre os demais, a grande vantagem de ser empregado como anestésico de ação média.

Verificamos, na bibliografia consultada, que o pentobarbital sódio, anestésico de ação curta, vem sendo utilizado com sucesso em medicina veterinária, num grande número de operaçōes.

Sendo o referido agente pouco conhecido e pouco aplicado em nosso país como anestésico, procuramos averiguar a possibilidade de empregá-lo em nossas intervenções cirúrgicas. 
Assim, dois motivos nos levaram a confecção dêste trabalho: a falta de uma apresentação comercial para uso veterinário e a feitura de uma solução única.

Utilizamos em nossos trabalhos o produto farmacêutico destinado à medicina humana, para aplicação como sedativo por via oral e apresentado sob forma de cápsulas de gelatina.

Ao iniciarmos nossas observações, pensamos nas dificuldades que poderiam surgir com um sal assim acondicionado e de uso diferente do da via intravenosa; entretanto, diante dos resultados obtidos, com esta solução anestésica resolvemos estudar detalhadamente sua aplicação.

\section{I - ADMINISTRAÇÃO DO PENTOBARBITAI. SODIO}

$\mathrm{Na}$ administração intravenosa do pentobarbital, seguimos a rotina utilizada na aplicação das anestesias gerais:

1) Prepuro do animal.

Salienta-se a necessidade do estado de jejum do doente.

2) Preparo da solução.

$O$ pentobarbital se apresenta no comércio sob a denominação de "nembutal", em cápsulas de $0,10 \mathrm{~g}$ do sal, as cápsulas são acondicionadas em vidros de 10 ou 25 unidades.

E um sal finamente pulverizado, branco, amorfo, inodoro, soluvel na água, de preferência a quente.

A soluçāo aquosa é límpida, transparente e pouco estável, o que nos impõe a necessidade de prepará-la no momento do uso.

Deve-se despresar as soluções com grumos ou aspecto opalescente.

Iniciamos nossas experiências com soluções variando de 1,5 a $3 \%$, e pelos resultados verificados foi esta última concentração $(3 \%)$ a que melhor comportamento demonstrou fornecendo ótimo periodo de anestesia cirúrgica.

A solução, como dissemos mais acima, é preparada no momento do uso, e procuramos realizá-la do modo mais rápido e simples.

Agimos da seguinte maneira:

a) esteriliza-se pela fervura um tubo de ensaio

b) colocam-se $10 \mathrm{~cm}^{3}$ de água distilada e esterilizada, aquecendo-se no momento, na própria água fervente que serve para esterilizar a seringa e o tubo de ensáio

c) dissolve-se uma por vez o conteúdo de três cápsulas e sempre aquecendo até a dissolução completa 
d) resfria-se

e) injeta-se.

3) Técnicu de administração.

A injeção intravenosa da solução anestésica de pentobarbital é feita pouco antes ou no momento em que a operação está para ser iniciada.

Injetamos de maneira bastante lenta, segundo a técnica de Wright e OYLER, isto é, $2 / 3$ da dose num espaço de trinta segundos e o restante após intervalo de um minuto.

Sòmente depois de se ter injetado a dose inicial, nota-se que o animal cai em sonolência e raramente pratica movimentos de reaçāo.

Retira-se a mordaça, e após o intervalo de um minuto, injeta-se o restante.

Com referência à quantidade e como dissemos, na parte anterior, usamos sempre uma solução a $3 \%$, portanto, cada $10 \mathrm{~cm}^{3} \mathrm{com}$ $0,30 \mathrm{~g}$ do sal e da qual administramos $1 \mathrm{~cm}^{3}$ por quilo de pêso vivo; é portanto, indispensável a pesagem do animal.

$$
\text { II - MARCHA DA ANESTESIA }
$$

De um modo geral e baseados nas nossas verificações, os vários estadios conhecidos nas anestesias completas. assim se apresentam durante aquela praticada pelo pentobarbital.

A indução é rápida e fácil, sem excitação, nem rigidez, com relaxamento muscular completo.

A imanutenção apresenta um tempo ótimo de anestesia cirúrgica, isto é. variando entre uma hora e meia a duas horas.

O despertar é demorado, algumas vêzes com excitação e normalmente sem vômitos. Apresenta-se com as duas fases bem características das anestesias gerais: inicialmente retôrno dos reflexos e posteriormente da consciência. Após a anestesia cirúrgica pròpriamente dita, obtem-se sempre um estado de anestesia incompleta, muito rantajosa para o animal operado.

Quando se administra, por acaso uma dóse insuficiente, o animal é tomado de uma excitação, grita, late e torna-se agressivo. Neste casso, podemos completar a anestesia com a própria solução de pentobarbital ou com éter.

Pará se apreciar o grau de anestesia, elaboramos um quadro de "sinais clínicos" observando as principais modificações funcionais que sofre o animal quando submetido à anestesia pelo pentobarbital. 
Entre os sinais clínicos temos: a temperatura diminui sensìvelmente, o número de pulsações aumenta e os movimentos respiratórios diminuem (respiração superficial).

Dos sinais clínicos particulares destacamos:

1. perda rápida de consciência

2. movimentos do torax e ruídos respiratórios diminuidos

3. amplitude respiratória aumentada

4. colorido normal das mucosas visíveis

5. abertura palpebral persistente (semi-aberta)

6. reflexos conjuntivos palpebral e corneal desaparecidos

7. o globo ocular permanece fixo, completamente imóvel, reluzente e úmido

8. pupila contraída

9. relaxamento muscular completo com silêncio abdominal profundo.

\section{III - ACIDENTES E MEDIDAS DE PREVENCĀO}

A morte pelo pentobarbital resulta da super dosagem, apezar de apresentar margem de segurança superior aos outros barbituratos.

$O$ acidente de maior freqüência é a paralisação respiratória e o seu quadro completo já é bastante conhecido.

$\mathrm{Na}$ tentativa de combater êste acidente, usamos vários estimulantes: coramina, lobelina, cardiazol. Tem sido aconselhado também a picrotoxina e a analepsina.

Outros meios que não devemos despresar são: respiração artificial, oxigênio ou mistura de oxigênio e anhidrido carbônico.

$$
\text { IV - INDICAÇ̋ES }
$$

A anestesia pelo pentobarbital tem provado ser útil nas intervenções de longa duração.

Seria desnecessário fazer uma relação, porque este tipo de anestesia é sòmente contraindicado em animais com moléstias que diminuem a amplitude respiratória e nos casos de fraqueza com mau estado geral.

Em relação à idade, aplicamos sem acidentes em cães desde dois meses até em idade avançada; em um de nossos casos o paciente tinha aproximadamente 15 anos (observação $n .^{\circ} 6$ )

$$
\mathrm{V} \text { - VANTAGENS }
$$

A rapidez, com que se obtem o periodo de manutenção neste tipo de anestesia com ausência completa de excitação torna-o superior ao de inhalação. 
Como vantagens secundárias temos: pouca toxicidade, facilidade de preparação, de aquisição e de administração.

Ausência de vômitos.

Ampla margem de segurança.

Nũo é inflamável, nem explosivo.

\section{VI - OBSERVAÇõES}

No decurso de nossos trabalhos contamos já com aproximadamente cincoenta casos de anestesia pelo pentobarbital, sem um único acidente; dêstes casos escolhemos para ilustração do presente trabalho apenas onze, cujos detalhes vão a seguir:

Observação N.’ 1

Sexo: macho. Raça: não identificável. Idade: 士 1 ano. Pêso: $8 \mathrm{~kg}$ e meio. Dose: $8,5 \mathrm{~cm}^{3}=0,255 \mathrm{~g}$.

\begin{tabular}{|c|c|c|c|c|c|c|c|}
\hline & & & & & Pulso & Trmperatura & Mov. respiratúrios \\
\hline Inicial & . & . & . & . & 110 & 3808 & 24 \\
\hline 5 minutos & . & . & . . & . & 140 & $38^{\circ} 3$ & 20 \\
\hline 15 & . & . & . & . & 130 & $37^{\circ} 2$ & 16 \\
\hline 30 &.$\quad$. & . & . & . & 140 & $37^{\circ} 0$ & 10 \\
\hline 60 & . $\quad$. & . & . . & . & 112 & $36^{\circ} 0$ & 12 \\
\hline
\end{tabular}
Despertar: após 9 horas.

24 horas

Fulso

110
Temperatura Mov. respiratórios $399^{\circ} 0 \quad 40$

Observação N.๑2

Sexo: macho. Raça: Fox-terrier pêlo liso. Idade: \pm 3 anos.

Pêso: $9 \mathrm{~kg}$ e meio. Dose: $9,5 \mathrm{~cm}^{3}=0,285 \mathrm{~g}$.

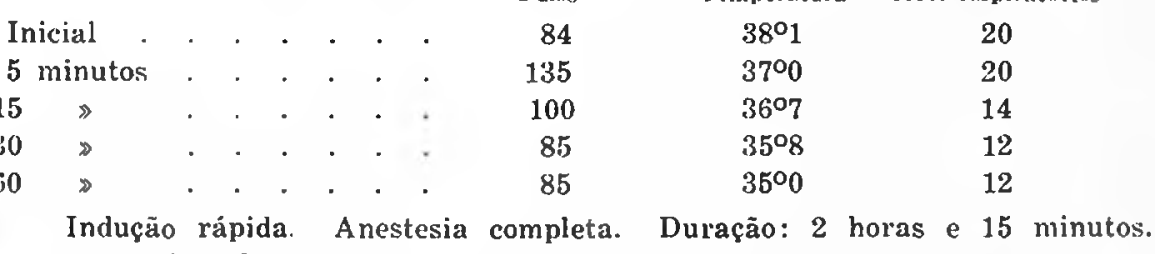

Despertar: após 8 horas.

24 horas. . . . . 95

$$
\begin{array}{ccc}
\text { Pulso } & \text { Temperatura } & \text { Mov. respiratórios } \\
95 & 38^{\circ} 8 & 18
\end{array}
$$

Observação N. ${ }^{\circ} 3$

Sexo: macho. Raça: cruzamento $/$ policial. Idade: $\mp 5$ anos.

Pêso: $9 \mathrm{~kg}$ e meio. Dose: $9,5 \mathrm{~cm}^{3}=0,285 \mathrm{~g}$.

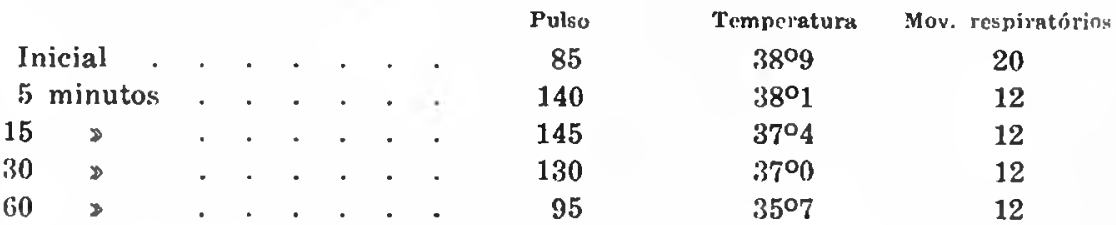


Indução rápida. Anestesia completa. Duração: 2 horas c 5 minutos. Despertar: após 7 horas.

24 horas . . . . . . . . . $\quad \begin{array}{cccc}\text { Pulso } & 92 & \text { Temperatura } & \text { Mor. respiratório: } \\ \end{array}$

\section{Observação N.0 4}

Scxo: macho. Raça: não identificávcl. Idade: 10 meses Pêso: $6 \mathrm{~kg}$. Dose: $6 \mathrm{~cm}^{3}=0,180 \mathrm{~g}$.

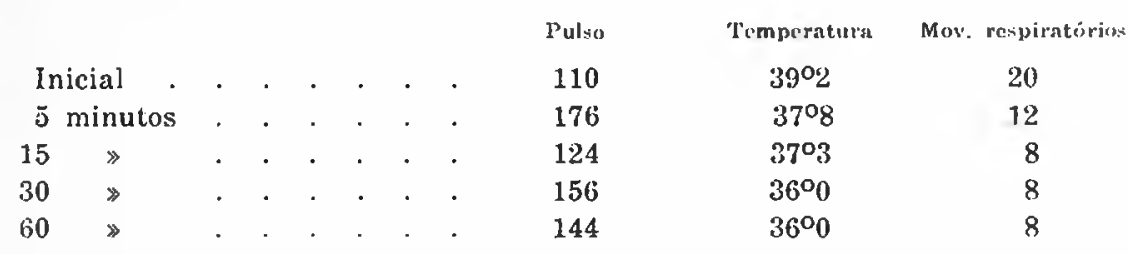

Indução rápida. Anestesia completa. Duração: 1 hora e 30 minutos. Despertar: após 8 horas.

$\begin{array}{ccccc} & \text { Pulso } & \text { Temperatura } & \text { Mos. respiratínios } \\ 24 \text { horas . . . . . . . . . } & 110 & 3901 & 20\end{array}$

Observação N:"5

Sexo: macho. Raça: não identificável. Idade: \pm 6 anos.

Pêso: $11 \mathrm{~kg}$ e meio. Dose: $11,5 \mathrm{~cm}: 3=0,345 \mathrm{~g}$.

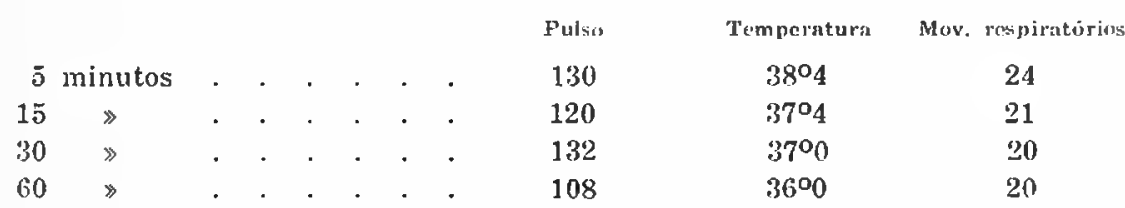

Indução rápida. Anestesia completa. Duração: 1 hora e 80 minutos. Despertar: após 7 horas e meia.

$2 \cdot 1$ horas

Pulso

92
Temperstura Mov. respiratiolins $38^{\circ} 2$
24

\section{Observação N.o 6}

Sexo: fêmea. Raça: Lulú. Idade: 15 anos.

Pêso: $9 \mathrm{~kg}$ e meio. Dose: $9,5 \mathrm{~cm}^{3}=0,285 \mathrm{~g}$.

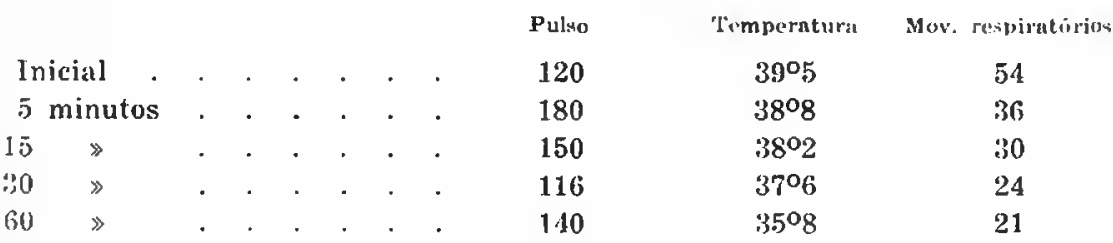

Indução rápida. Anestesia completa. Duração: 2 horas c 40 minulos. Despertar: após 8 horas. Operação: Ovariectomia com retirada de 1 quisto ovárico. Animal novamente anestesiado com intervalo de 1 mês e meio. 


\section{Observação N. ${ }^{\circ} T$}

Scxo: fêmea. Raça: nāo identificável. Idade: 1 ano.

Pêso: $15 \mathrm{~kg}$. Dose: $15 \mathrm{~cm}^{8}=0,450 \mathrm{~g}$.

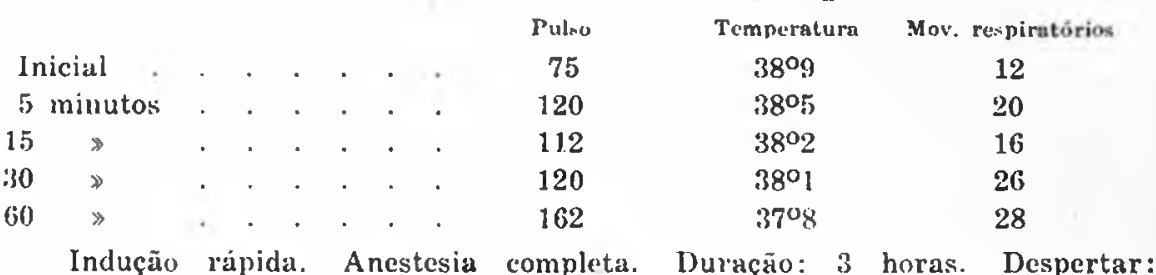
após 10 horas. Operação: Ovariectomia.

Observação N.० 8

Sexo: fêmea. Raça: não identificável. Idade: 3 meses Pêso: $2 \mathrm{~kg}$ e meio. Dose: $2 \mathrm{~cm}^{3}$ e meio $=0,075 \mathrm{~g}$.

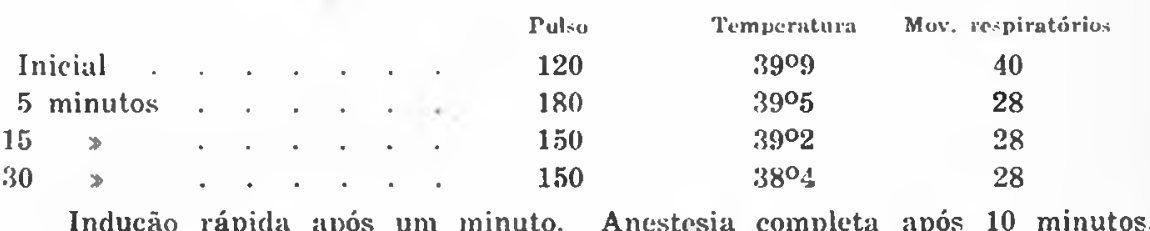
Duração: 1 hora. Despertar: após 4 horas.

Observação N.o9

Sexo: macho. Raça: cruzamento e/ basset. Idacle: \pm 1 ano e meio. Pêso: $8 \mathrm{~kg}$ e meio. Dose: $8,5 \mathrm{~cm}$ i. Solução: $2 \%=0,170 \mathrm{~g}$.

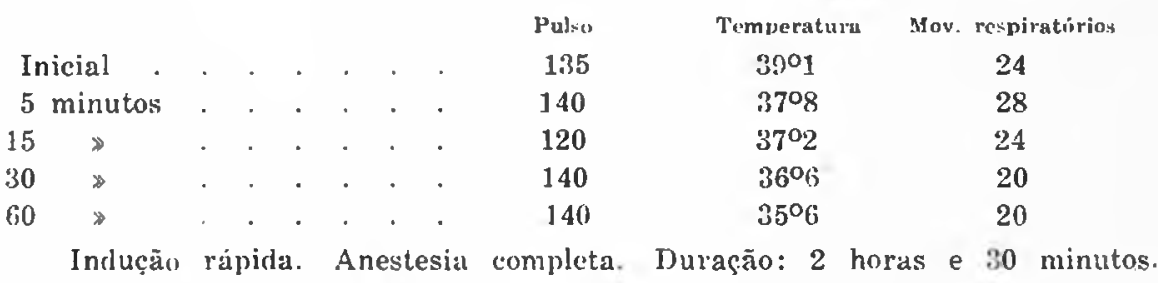
Despertar: após (i horas e :35 minutos.

Este animal recebeu como pré-anestesia $2 \mathrm{~cm}^{3}$ de atropomorfina. Observacão N. 10

Sexo: macho. Raça: não identificável. Idade: \pm 10 anos.

Pêso: $7,0 \mathrm{~kg}$. Dose: $7 \mathrm{~cm}^{3}$. Solução: $1,5 \%=0,105 \mathrm{~g}$.

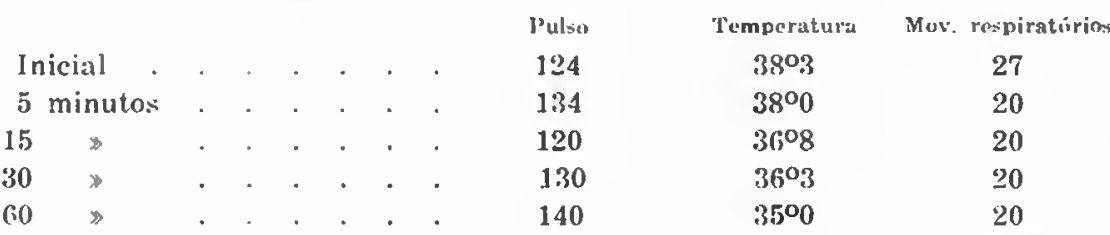

Despertar: 3 horas e 30 minutus. Anestesia incompleta.
24 horas apos
$38^{\circ} 2$
20

l'ulso

Temperatura

Mow. respiratiorios

120 
Observação N. 11

Sexo: macho. Raça: cruzamento policial. Idade: t上 3 anos.

Pêso: $9 \mathrm{~kg}$ e meio. Dose: $9 \mathrm{~cm}^{3}$ e meio. Solução: $2 \%=0,190 \mathrm{~g}$.

Recebeu mais $3 \mathrm{~cm}$ " após 10 minutos da mesma solução e $1 \mathrm{~cm}$ " e meio de atropomorfina. Indução rápida. Anestesia incompleta. Duração: 2 hor'as e 30 minutos com auxílio de mascara com éter.

\section{RESUMO}

Os A. A. estudam a anestesia geral intravenosa pelo pentobarbital (nembutal) no cão.

Determinam processo de preparação fácil, utilizando o produto farmacêutico destinado à medicina humana, para aplicação como sedativo por via oral e apresentado sob forma de cápsulas de gelatina.

Utilizam solução aquosa única à $3 \%$, administrada de acôrdo com o pêso do animal ( $1 \mathrm{~cm}^{3}$ por quilo de pêso vivo).

Dentre cerca de cincoenta casos já estudados apresentam dados detalhados de 11 (onze) dos quais 3 (três) com pré-anestesia (atropomorfina) e concluem sôbre os principais sinais clinicos e a margem de segurança dêsse anestésico.

\section{SUMMARY}

The AA. study the intravenous general anesthesia by the pentobarbital (nembutal) in the dog.

Utilising the pharmaceutical product employed in hwman medicine as sedative and presented in pulvules for oral use.

They describe a simple process to make it profitable to intravenous administration.

A \& per cent aqueous solution of the medicament is administered proporcional to the animal body weight ( $1 \mathrm{~cm}^{3}$ per kilogram.)

Among over fifty studied cases they present detailed data of eleven ones, 3 of them with pre-anesthesia (atropomorphine) and establish the main clinical signs and the security margin of the anesthetic.

\section{BIBLIOGRAFIA}

Adams, R. C. - 1944 - Intravenous anesthesia. New York Paul B. Hoebar, Inc. FETHERS, G. - 1938 - Anaesthesia in small animals. Aust. Vet. Jour., 14: 142-6 GoOdman, L. - Gilman, A. - 1941 - The pharmacological basis of Therapeutics. New York, The Macmillan Company

Matera, E. A. - Tabarelli, J. F. - 1945 - Contribuição ao estudo da anestesia intravenosa pelo Thionembutal no cāo. Adaptação do método fracionado de Lundy. These apresentada ao III Congresso Brasileiro de Medicina Veterinária Pôrto Alegre 
E. A. Matera e O. A. Castrignano - Anestesia intravenosa no cão

Milks, H. J. - 1943 - Practical Veterinary Pharmacology, Materia Medica and Therapeutics. 5th. ed. Chicago, Alexander Eger

Mosty, H. J. - 1941 - Problems of anaesthesia in small animals. Vet. Med., $36: 144-54$

RitchiE, A. F. - $1938-A$ case series from general practice. III: In the dog. Vet. Rec., 50: 158-62

Sweebe, E. E. - 19:36 - Anesthesia. Vet. Med., 31: 158-62

SwEEBE, E. E. - 1938 - Barbituratesas safe and efficient general anesthetics in canine surgery. Jaur. Amer. Vet. Ass, 45 N. S.: 145-51

WooldridGE, G H. - 1934 - Encyclopaedia of Veterinary medicine, surgery and obstetrics. 2nd. ed. 2 (2) : 1279-81. London, Oxford University Press

WRIGHT - OYLER - 1934 - L'anesthésis du chien par le nembutal. Vet. Rec. "in" Rer. Vét. el Jour. Méd. Vét., 88: 572-4, 1936

WRIGHT, J. G. - 1942 - Veterinary anaesthesia: 164-82. London, Baillière, Tindall \& Cox 